\title{
Comparison of Stemness and Metastasis Genes in Breast Cancer and Gastric Cancer
}

\author{
Mahnaz Azimi 1, 2, Mahsa Rahimi ${ }^{2,3}$, Marzieh Ebrahimi ${ }^{2, *}$, Javad \\ Firouzi $^{2}$, Parisa Sahranavard ${ }^{2}$ \\ ${ }^{1}$ Department of Genetics, University of Science and Culture, ACECR, Tehran, Iran \\ ${ }^{2}$ Department of Stem Cells and Developmental Biology at Cell Science Research \\ Center, Royan Institute for Stem Cell Biology and Technology, ACECR, Tehran, Iran \\ ${ }^{3}$ Faculty of Advanced Medical Sciences, Tabriz University of Medical Sciences, Tabriz, \\ Iran \\ ${ }^{*}$ Corresponding author: Marzieh Ebrahimi, Department of Stem Cells and \\ Developmental Biology at Cell Science Research Center, Royan Institute for Stem Cell \\ Biology and Technology, ACECR, Tehran, Iran.E-mail: mebrahimi@royaninstitute.org
}

DOI: $10.21859 / \mathrm{mci}$-supp-11

\section{Keywords:}

CSCs

Metastasis

Gene

Stemness

Metastasis

\begin{abstract}
Introduction Cancer stem cells (CSCs) are a major cause of tumor metastasis and recurrence. On the other hand, Stemness and metastasis process are the most common cause of mortality in breast cancer and gastric cancer. Therefore, in this study by using literature review, 10 genes were selected which are able to regulate any of these two properties in both cancers. Then by comparing them, we candidated key biomarkers which were common in both cancers.
\end{abstract}

Materials and Methods: We searched most common stemness and metastasis genes by literature review and 10 genes were selected. Then, we cultured MCF-7 cell line of breast cancer and MKN-45 cell line of gastric cancer to form sphere in serum-free medium. The culture environment is important in maintaining CSCs in vitro and sphere formation is one of the methods for culturing CSCs. Then we evaluated the expression of these 10 genes on sphere cells using qRT-PCR in MCF-7 cell line of breast cancer and MKN-45 cell line of gastric cancer and compared them with parental cells which were adherent cells. After that, we compared the results of qRT-PCR of those two cell lines together.

Results: The results indicated that 4 stemness genes including OCT4, NOTCH, SOX2, CDH1 were up-regulated and some metastasis genes include TWIST1 and SNAIL1 were up-regulated and SNAIL2 was down regulated in both cell lines.

Conclusions: Sphere-forming cells showed that they were positive for cancer stem cells and metastatic markers, so sphere forming culture is a suitable method for growing CSCs that can induce the expression of set of candidate stemness and metastasis genes. 